\title{
Photorefractive properties of lithium niobate crystals doped with manganese
}

\author{
Yunping Yang and Demetri Psaltis \\ Department of Electrical Engineering, California Institute of Technology, Pasadena, California 91125
}

Marc Luennemann, Dirk Berben, Ulrich Hartwig, and Karsten Buse

Universität Bonn, Physikalisches Institut, Wegelerstr. 8, D-53115 Bonn, Germany

Received October 12, 2002; revised manuscript received March 3, 2003

\begin{abstract}
The photorefractive properties of lithium niobate crystals doped with manganese (Mn) have been investigated. It is found that the effect of dark decay due to electron tunneling, which is the limiting factor of the highest practical doping level, is less in $\mathrm{LiNbO}_{3}: \mathrm{Mn}$ than in $\mathrm{LiNbO}_{3}: \mathrm{Fe}$, and higher doping levels can be used in $\mathrm{LiNbO}_{3}: \mathrm{Mn}$ to achieve larger dynamic range and sensitivity for holographic applications. The highest practical doping level in $\mathrm{LiNbO}_{3}: \mathrm{Mn}$ has been found to be $\sim 0.5 \mathrm{wt} . \% \mathrm{MnCO}_{3}$, and refractive-index changes and sensitivities up to $1.5 \times 10^{-3}$ and $1.3 \mathrm{~cm} / \mathrm{J}$ are measured for extraordinarily polarized light of the wavelength $458 \mathrm{~nm}$. It has been found that, in terms of both dynamic range (or refractive-index change) and sensitivity, the optimal oxidation state is highly oxidized. The distribution coefficient of $\mathrm{Mn}$ has been determined to be $\sim 1$. Absorption measurements are used to obtain more information about charge-transport parameters. The material is excellently suited for holographic recording with blue light. The hologram quality is outstanding because holographic scattering is much weaker compared with that in, e.g., iron-doped lithium niobate. Thermal fixing has been successfully demonstrated in $\mathrm{LiNbO}_{3}: \mathrm{Mn}$ crystals. (C) 2003 Optical Society of America

OCIS codes: $090.2900,090.7330,160.3730,210.0210,210.4810$.
\end{abstract}

\section{INTRODUCTION}

Holographic memories are of intense interest and have advantages compared with conventional memories in that they offer a data storage density of up to a few hundred gigabits per cubic centimeter through multiplexing and a data transfer rate of the order of gigabits per second with page access. ${ }^{1}$ Recently, a lot of research has been conducted on $\mathrm{LiNbO}_{3}$-based holographic storage systems, and several multiplexing methods have been proposed and demonstrated. ${ }^{1-6}$ For holographic storage systems, two of the most important system parameters are dynamic range $(M / \#)$ and sensitivity. ${ }^{7}$ The larger the $M / \#$, the higher the storage density and the better the signalto-noise-ratio. Sensitivity determines the recording speed. The higher the sensitivity, the shorter the recording time required. Also, it is found that the capacity of a holographic storage system is of the order of $V / \lambda^{3}$, where $V$ is the volume of the recording medium and $\lambda$ is the working wavelength, ${ }^{1}$ which means that higher storage density can be achieved by use of shorter wavelength. In general, it is desired to have a recording material with large $M / \#$ and high sensitivity and to work in the blue spectral region, where the wavelength is relatively short. In addition to the above desired properties, another one is the low holographic scattering, which will also result in better signal-to-noise ratio.

One approach to boosting the $M / \#$ and sensitivity for the $\mathrm{LiNbO}_{3}$-based holographic storage systems is to increase the doping level. Nevertheless, it has been found that there is a limit on the highest practical doping level of $\mathrm{LiNbO}_{3}$, and any further increase of the doping level above this limit cannot result in larger $M / \#$ and sensitivity. ${ }^{8}$ For example, in the widely used $\mathrm{LiNbO}_{3}: \mathrm{Fe}$ crystals, the highest practical doping level is $\sim 0.1$ wt. $\% \mathrm{Fe}_{2} \mathrm{O}_{3}$ and is found to be limited by a fast dark decay. ${ }^{8-11}$ It is desired to have highly doped crystals with acceptable dark decay.

Two dark-decay mechanisms have been identified in $\mathrm{LiNbO}_{3}$ crystals: proton compensation and electron tunneling. ${ }^{9,10}$ In general both, proton compensation and electron tunneling, contribute to the dark decay in $\mathrm{LiNbO}_{3}$ crystals. In crystals with low doping levels, proton compensation dominates the dark decay, and the time constant of this type of dark decay is inversely proportional to the proton concentration. If the dark decay is due to proton compensation, we can always slow down the dark decay by reducing the proton content. For crystals with high doping levels, e.g., 0.25 wt. $\% \mathrm{Fe}_{2} \mathrm{O}_{3}$, electron tunneling dominates the dark decay. This type of dark decay also limits the highest practical doping level in $\mathrm{LiNbO}_{3}$ crystals in, e.g., holographic storage systems and optical narrow-band wavelength filters. It is desirable to have crystals with high doping levels and the dark decay still dominated by proton compensation, that is, to reduce the effect of electron tunneling in highly doped lithium niobate crystals.

Electron tunneling is characterized by the dopant, and the probability of electron tunneling through a square potential barrier is

$$
p=\frac{16 E(V-E)}{V^{2}} \exp \left[-\frac{4 \pi d}{h} \sqrt{2 m(V-E)}\right],
$$


where $h$ is the Planck's constant, $m$ is the mass of the particle, $E$ is the energy of the particle, $V$ is the height of the barrier, and $d$ is the width of the barrier. Increasing the doping levels is equivalent to decreasing the average distance between nearby dopant sites, which can be considered as the width of the barrier of electron tunneling. From Eq. (1), we can see that the probability of electron tunneling is strongly dependent on the height of the barrier. The higher the barrier, the smaller the probability of electron tunneling. By using a dopant with a deeper energy level in lithium niobate crystals, we can increase the height of the barrier of electron tunneling and therefore decrease the effect of electron tunneling. It is known that the Mn center is deeper than the Fe center; thus the height of the barrier of Mn center should be larger than that of the $\mathrm{Fe}$ center. ${ }^{12}$ We expect that the electrontunneling effect in $\mathrm{LiNbO}_{3}: \mathrm{Mn}$ crystals is smaller. Therefore it is possible to use manganese-doped lithium niobate crystals with higher doping levels for holographic storage to get larger $M / \#$ and sensitivity. In our previously published paper, we have shown that the darkdecay time constants of nonfixed holograms in $\mathrm{LiNbO}_{3}$ crystals doped with 0.2 at.\% $\mathrm{Mn}$ at room temperature are several orders of magnitude larger than those of $\mathrm{LiNbO}_{3}: \mathrm{Fe}$ crystals with comparable doping levels. ${ }^{11}$ Recently we found, experimentally, that the highest practical doping level in manganese-doped lithium niobate for holographic storage is $\sim 0.5 \mathrm{wt} . \% \mathrm{MnCO}_{3}$. The largest $\mathrm{M} / \#$ and sensitivity measured in these crystals are 7.3 for a $0.84-\mathrm{mm}$-thick crystal and $1.3 \mathrm{~cm} / \mathrm{J}$ with the light wavelength $458 \mathrm{~nm}$ and extraordinary polarization. The optimal oxidation state of these crystals is highly oxidized. Moreover, some other advantages of manganese-doped lithium niobate crystals for holographic storage, such as excellent recording stability and repeatability as well as low holographic scattering, have been observed.

In order to tailor the material parameters of Mn-doped lithium niobate crystals for applications such as holographic storage, a quantitative description of the photorefractive properties is needed. Compared with Fe-doped lithium niobate crystals, much less is known about Mndoped lithium niobate, and only a few papers have addressed the photorefractive properties of Mn-doped lithium niobate. ${ }^{11-18}$

In this work, the photorefractive properties of $\mathrm{LiNbO}_{3}: \mathrm{Mn}$ crystals have been investigated. Material parameters, such as the distribution coefficient, are determined. Absorption measurements are used to obtain some information about several charge-transport parameters. The dynamic range $(M / \#)$ and sensitivity for crystals of different doping levels, different oxidation states, and for different light polarizations have been measured.

\section{FUNDAMENTALS}

One of the most important system metrics for holographic storage systems is the dynamic range $(M / \#)$. When $M$ holograms are multiplexed with the appropriate recording schedule, the diffraction efficiency of each hologram $(\eta)$ is given $b y^{7}$

$$
\eta=\left(\frac{M / \#}{M}\right)^{2} .
$$

The diffraction efficiency is the ratio of diffracted and incident light intensities. Equation (2) suggests that in multiplexing $M$ holograms with a prescribed diffraction efficiency $\eta$, increasing the $M / \#$ results in increasing the number of holograms that can be multiplexed, thus increasing the capacity of the holographic storage system. On the other hand, with a fixed number of holograms multiplexed, larger $M / \#$ results in higher diffraction efficiency for each hologram and therefore higher signal-tonoise-ratio and data transfer rate.

Another important system metric for a holographic storage system is sensitivity $S$, which determines the recording speed. The larger the sensitivity, the faster we can record the hologram with a fixed recording intensity.

Dynamic range $(M / \#)$ and sensitivity $(S)$ can be measured by single-hologram recording and erasure experiments. ${ }^{7}$ From the single-hologram recording and erasure curve, we can calculate $M / \#$ and $S$ using

$$
\begin{gathered}
M / \#=\left.\frac{\mathrm{d} \sqrt{\eta} \mid}{\mathrm{d} t}\right|_{t \ll \tau_{e}} \tau_{e}, \\
S=\frac{\mathrm{d} \sqrt{\eta} /\left.\mathrm{d} t\right|_{t \ll \tau_{e}}}{I L},
\end{gathered}
$$

where $\tau_{e}, I$, and $L$ are erasure time constant, total incident recording intensity, and the crystal thickness, respectively. Both $M / \#$ and sensitivity are proportional to the refractive-index change $\Delta n$, which is related to the space-charge field $E_{\mathrm{sc}}$ as

$$
\Delta n=-\frac{1}{2} \gamma_{\mathrm{eff}} n_{\mathrm{eff}}^{3} E_{\mathrm{sc}},
$$

where $\gamma_{\text {eff }}$ is the effective electro-optic coefficient, and $n_{\text {eff }}$ is the effective refractive index. ${ }^{19}$

Using the coupled-wave theory, we can obtain the diffraction efficiency of the volume hologram of symmetric transmission geometry as

$$
\eta=(1-R)^{2} \exp \left(-\frac{\alpha L}{\cos \theta}\right) \sin ^{2}\left(\frac{\pi L \Delta n}{\lambda \cos \theta}\right),
$$

where $R$ is the reflectivity, $\alpha$ is the intensity absorption coefficient, $L$ is the thickness of the crystal, $\theta$ is the incident angle inside the crystal, $\lambda$ is the wavelength outside the crystal, and $\Delta n$ is the amplitude of refractive-index change. ${ }^{20}$ The refractive-index change can be calculated from the measured diffraction efficiency.

Several charge-transport models have been developed to explain the photorefractive phenomenon, among which the one-center model, two-center model, and threevalence model are a few. The one-center model was developed in the early days of research on the photorefractive effect. The first complete set of partial differential equations of the one-center model was introduced by Kukhtarev et al. ${ }^{21}$ The predictions of the one-center model are in excellent agreement with experimental results obtained with $\mathrm{LiNbO}_{3}: \mathrm{Fe}$ and $\mathrm{LiNbO}_{3}: \mathrm{Cu}$ crystals 
at continuous-wave intensities. ${ }^{22-25}$ Using the firstorder approximation, we can analytically solve the Kukhtarev equations, which describe the photorefractive effect, to obtain the amplitude of the space-charge field for the short-circuited crystal $E_{\mathrm{sc}}$ as the following:

$$
E_{\mathrm{sc}}=E_{q} \sqrt{\frac{E_{\mathrm{ph}}^{2}+E_{\mathrm{d}}^{2}}{\left[\left(N_{A} / N_{D}\right) E_{\mathrm{ph}}\right]^{2}+\left(E_{\mathrm{d}}+E_{\mathrm{q}}\right)^{2}}},
$$

where $N_{D}$ and $N_{A}$ are the total concentration of the deep traps (i.e., $\mathrm{Fe}$ ) and the concentration of the ionized deep traps (i.e., $\mathrm{Fe}^{3+}$ ), respectively. ${ }^{19,21}$ In these equations, the saturation field $E_{\mathrm{q}}$, the bulk photovoltaic field $E_{\mathrm{ph}}$, and the diffusion field $E_{\mathrm{d}}$ are given by

$$
\begin{aligned}
E_{\mathrm{q}} & =\frac{q N_{A}\left(N_{D}-N_{A}\right)}{\epsilon K N_{D}}, \\
E_{\mathrm{ph}} & =\frac{p r_{A} N_{A}}{q \mu s}, \\
E_{\mathrm{d}} & =\frac{k_{B} T}{q} K,
\end{aligned}
$$

with $\epsilon, r_{A}, s, p$, and $\mu$ being the dielectric constant, the recombination rate of electrons from the conduction band, absorption cross section for the excitation of electrons from the deep traps to the conduction band, the bulk photovoltaic constant of the deep traps (both at the recording wavelength), and the charge-carrier mobility, respectively. Electron charge, Boltzmann constant, and absolute temperature are represented by $q, k_{B}$, and $T$, respectively. The magnitude of the $\mathbf{K}$ vector is denoted by $K$.

\section{SAMPLES AND EXPERIMENTAL METHODS}

The photorefractive properties of several Mn-doped $\mathrm{LiNbO}_{3}$ crystals with different doping levels are investigated. Table 1 shows some material parameters of these samples. The dimensions of the samples are defined as $a \times b \times c$, where $c$ is the length along the $c$ axis and $b$ is the thickness. Thermal treatments were used to change the oxidation state of some of the samples. The temperature, duration, and atmosphere of thermal treatment are controlled to obtain the desired oxidation state.

Two key parameters of photorefractive lithium niobate crystals are dopant and doping level. Knowing the actual doping level in the crystal is the starting point for all investigations of photorefractive properties. Usually, a certain amount of transition-metal dopants, such as Fe, $\mathrm{Cu}, \mathrm{Ce}$, and $\mathrm{Mn}$, are added to the melt as oxides during the growth of crystals. The nominal doping level is measured in weight percentage to the mixture of $\mathrm{Li}_{2} \mathrm{O}$ and $\mathrm{Nb}_{2} \mathrm{O}_{5}$ or in mole percentage to $\mathrm{LiNbO}_{3}$. In some cases, the actual doping level inside lithium niobate crystals is different from the nominal doping level. The ratio between the actual doping level and the nominal doping level is called the "distribution coefficient". In order to measure the distribution coefficient of manganese-doped lithium niobate, several samples with different nominal doping levels, $0.05,0.1,0.2,0.5$, and $1.0 \mathrm{wt} . \% \mathrm{MnO}$, have been investigated. Two different methods were applied for all the crystals by independent parties. One method is neutron activation analysis, ${ }^{26}$ the other is inductively coupled plasma optical emission spectroscopy. ${ }^{27}$ The method of neutron activation analysis yields a distribution coefficient of 1.1, while the method of inductively coupled plasma optical emission spectroscopy yields a distribution coefficient of $\sim 0.85$. The average distribution coefficient obtained from these two methods is $\sim 1$. Considering all the possible uncertainties, it is reasonable to take the distribution coefficient of manganese-doped lithium niobate crystals as 1 .

In our experiments of measuring $M / \#$ and sensitivity, an argon-ion laser beam with the wavelength $458 \mathrm{~nm}$ was used to record and to erase holograms. The crystal was placed on a rotation stage. The laser beam was split into two equal-intensity beams. The grating vector was always aligned along the $c$ axis. During recording, one beam was blocked from time to time to measure the holographic diffraction efficiency as the ratio of diffracted and incident light intensities. We used Bragg-mismatched erasure, i.e., during erasure the sample was rotated far away from the Bragg-matched position (by at least 50 times of the selectivity) and illuminated by the same two beams that were used to record holograms. This guaranteed that the spurious gratings recorded during erasure would have little effect on the measurement of $M / \#$ and sensitivity. Moreover, in order to avoid building strong spurious holograms and fanning as well as the nonuniform erasure due to the interference pattern inside the samples, the sample was rotated 0.02 deg every $10 \mathrm{~s}$ during erasure. At the end of each period of erasure, the diffraction efficiency was measured by scanning over an adequate range of angle (which covered the Bragg-matched position) and finding the maximum diffraction efficiency with only the reference beam on. $M / \#$ and sensitivity were calculated according to Eqs. (3) and (4). As an alternative, recording and erasure of the holographic gratings with the blue light (wavelength $458 \mathrm{~nm}$ ) was monitored with an additional ordinarily polarized red beam (wavelength $633 \mathrm{~nm}$ ). The red light yields a smaller diffraction efficiency because of the ordinary polarization

Table 1. Summary of Parameters of Mn-Doped Lithium Niobate Samples

\begin{tabular}{lcccc}
\hline Sample & Doping level & $\mathrm{C}_{\mathrm{Mn}}\left(\times 10^{24} \mathrm{~m}^{-3}\right)$ & Dimension $\left(\mathrm{mm}^{3}\right)$ & Oxidation State \\
\hline S1 & 0.20 wt.\% $\mathrm{MnO}$ & 78 & $15.0 \times 2.0 \times 20.0$ & As grown \\
S2 & 0.05 wt.\% $\mathrm{MnO}$ & 20 & $15.0 \times 2.0 \times 20.0$ & As grown \\
S3 & 0.50 wt.\% $\mathrm{MnO}$ & 196 & $15.0 \times 2.0 \times 20.0$ & As grown \\
S4 & 0.50 wt.\% $\mathrm{MnCO}_{3}$ & 122 & $4.0 \times 0.84 \times 5.0$ & Highly oxidized \\
S5 & 0.50 wt.\% $\mathrm{MnCO}_{3}$ & 122 & $4.0 \times 0.84 \times 5.0$ & Highly reduced \\
\hline
\end{tabular}


and the longer wavelength. However, we calibrated this difference based on experimental data and were in the following able to deduce $M / \#$ and recording sensitivity for blue light from the readout with red light. Several experiments were performed with both methods (reading with blue/red light), and the obtained $M / \#$ and sensitivity values were always in perfect agreement.

\section{EXPERIMENTAL RESULTS}

In this section the obtained absorption data, dark-decay time constants measured at different temperatures, dynamic ranges and sensitivity data, the results of thermal fixing, and holographic scattering data are presented.

\section{A. Absorption Measurements}

Absorption measurement is a very important method to characterize the material parameters of photorefractive lithium niobate crystals. Usually, the characteristic absorption band and absorption coefficient are used to calibrate the concentration of the corresponding absorption center. The absorption spectra of $\mathrm{LiNbO}_{3}: \mathrm{Mn}$ crystals in the wavelength range of $0.3-3 \mu \mathrm{m}$ were obtained with a Varian Cary-500 spectrometer. Figure 1 shows a typical absorption spectrum of one $\mathrm{LiNbO}_{3}: \mathrm{Mn}$ crystal, $\mathrm{S} 1$, for ordinary light polarization. The nominal doping level of sample $\mathrm{S} 1$ is 0.2 wt.\% $\mathrm{MnO}$; that is, the $\mathrm{Mn}$ concentration is $78 \times 10^{24} \mathrm{~m}^{-3}$, and the thickness of the crystal is $2 \mathrm{~mm}$ with the oxidation state as grown. There are three apparent absorption bands in the wavelength range of $0.3-3$ $\mu \mathrm{m}$. One is around $2.87 \mu \mathrm{m}$, which is the characteristic absorption band of $\mathrm{OH}^{-} .{ }^{28}$ This absorption band is independent of dopant and doping level and can be used to calculate the concentration of protons in $\mathrm{LiNbO}_{3}$ crystals. Another apparent absorption band is the wide band centered around $1.2 \mu \mathrm{m}$. Presumably, this is due to small polarons. ${ }^{29}$ The third apparent absorption band is around $577 \mathrm{~nm}$, and some researchers attributed this absorption to a crystal-field transition of $\mathrm{Mn}^{3+}$ or $\mathrm{Mn}^{4+} .14$

Figure 2 shows the absorption spectra of three $\mathrm{LiNbO}_{3}: \mathrm{Mn}$ crystals with different doping levels, S1-S3, for ordinary polarization in the wavelength range of 400 $\mathrm{nm}$ to $1500 \mathrm{~nm}$. The oxidation states of all the three

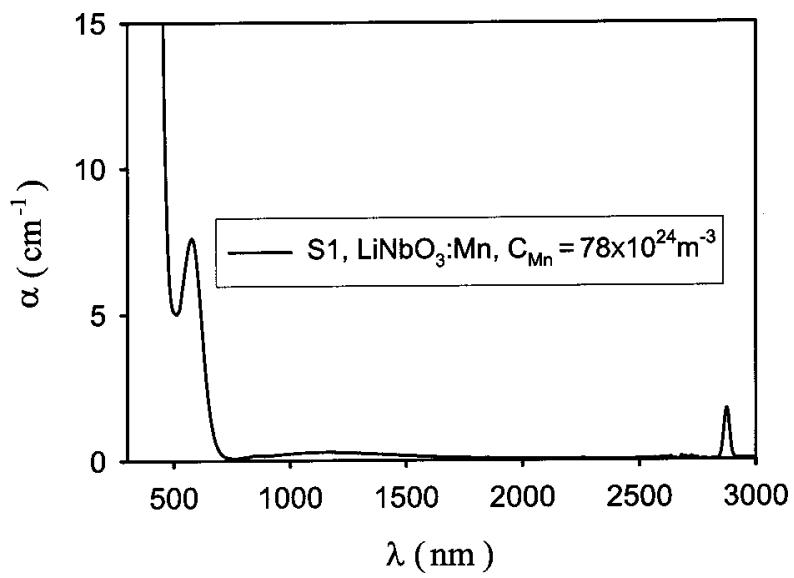

Fig. 1. Absorption coefficient $\alpha$ versus light wavelength $\lambda$ for an as-grown $\mathrm{LiNbO}_{3}: \mathrm{Mn}$ crystal for ordinary light polarization.

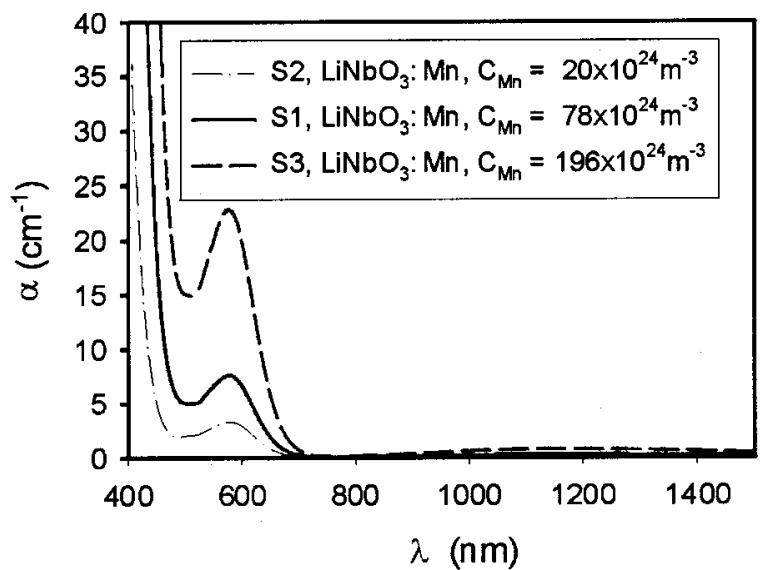

Fig. 2. Absorption coefficient $\alpha$ versus light wavelength $\lambda$ for three as-grown $\mathrm{LiNbO}_{3}: \mathrm{Mn}$ crystals with different doping levels for ordinary light polarization.

crystals are as grown. From Fig. 2 we can see that the absorption band around $577 \mathrm{~nm}$ of $\mathrm{LiNbO}_{3}: \mathrm{Mn}$ is related to the doping level; the higher the doping level, the larger the absorption coefficient at $577 \mathrm{~nm}$. Another observation from Fig. 2 is that the absorption edge, which is typically determined by the energy needed for band-to-band absorption, shifts from shorter wavelength to longer wavelengths with the doping level increasing. One possible explanation of this phenomenon is that there is another broad absorption band of $\mathrm{Mn}$ dopants in the range of wavelengths $300 \mathrm{~nm}$ to $500 \mathrm{~nm}$, and the shifting of the edge is the result of the combination of the band-to-band absorption and this broad absorption band. Anyhow, the absorption spectrum of $\mathrm{LiNbO}_{3}: \mathrm{Mn}$ crystals for wavelengths below $450 \mathrm{~nm}$ does not give much information about the photorefractive parameters of $\mathrm{LiNbO}_{3}: \mathrm{Mn}$.

The absorption band around $577 \mathrm{~nm}$ is very interesting. First, unlike the absorption band around $477 \mathrm{~nm}$ in $\mathrm{LiNbO}_{3}: \mathrm{Fe}$, which is due to the transition of electrons from $\mathrm{Fe}^{2+}$ to the conduction band and that can be used to calibrate the concentration of $\mathrm{Fe}^{2+}$, this absorption band around $577 \mathrm{~nm}$ in $\mathrm{LiNbO}_{3}: \mathrm{Mn}$ is not due to the transition of electrons from $\mathrm{Mn}^{2+}$ to the conduction band, because the more oxidized the $\mathrm{LiNbO}_{3}: \mathrm{Mn}$ crystal is, the higher the absorption peak of this band becomes. Figure 3 shows the spectra of two $\mathrm{LiNbO}_{3}$ crystals doped with 0.5 wt. $\% \mathrm{MnCO}_{3}, \mathrm{~S} 4$ and $\mathrm{S} 5$, which were cut from the same boule. S4 was highly oxidized, and S5 was highly reduced. We can see that the wavelengths of the absorption peaks of these two crystals are the same, around 577 $\mathrm{nm}$, with the absorption peak coefficient of the highly oxidized crystal much larger than that of the highly reduced. We can use the absorption coefficient of this band to quantify the oxidation state. Furthermore, this absorption band is not photorefractive, that is, the absorption at 577 nm will not help the photorefractive process. It is generally assumed that $\mathrm{Mn}$ traps exist in $\mathrm{LiNbO}_{3}$ as $\mathrm{Mn}^{2+}$ and $\mathrm{Mn}^{3+}$, or even as $\mathrm{Mn}^{4+}$. Since the more oxidized, the more absorptive around $577 \mathrm{~nm}$ it is, this absorption band around $577 \mathrm{~nm}$ could be related to the concentrations of $\mathrm{Mn}^{3+}$ or $\mathrm{Mn}^{4+}$.

Another interesting phenomenon about the absorption spectrum of $\mathrm{LiNbO}_{3}: \mathrm{Mn}$ is the large dichroism. The di- 
chroism is defined as the difference between the absorption coefficients for ordinary and for extraordinary light polarizations. Figure 4 shows the measured absorption spectra of one $\mathrm{LiNbO}_{3}: \mathrm{Mn}$ crystal, $\mathrm{S} 4$, for ordinary and extraordinary light polarizations. From Fig. 4 we can see that in the visible range, the absorption coefficient for ordinary polarization is larger than that for extraordinary polarization. Around the absorption edge, this dichroism is equivalent to a shift of the edge. Since the absorption edge is very steep, the dichroism around the absorption edge is very large even though the shift due to the dichroism is small. The dichroism of the absorption band around $577 \mathrm{~nm}$ is consistent with attribution to a crystalfield transition. The absorption peak of this band for extraordinary light shifts to shorter wavelength. Later we will show that the absorption of extraordinary polarization being less than that of ordinary polarization makes the optimal polarization for $\mathrm{LiNbO}_{3}: \mathrm{Mn}$ crystal extraordinary.

\section{B. Dark Decay in $\mathrm{LiNbO}_{3}: \mathrm{Mn}$}

In the measurements of the dark decay, the crystals were placed on a heatable plate whose temperature was controlled within $0.1^{\circ} \mathrm{C}$ accuracy. An argon-ion laser beam

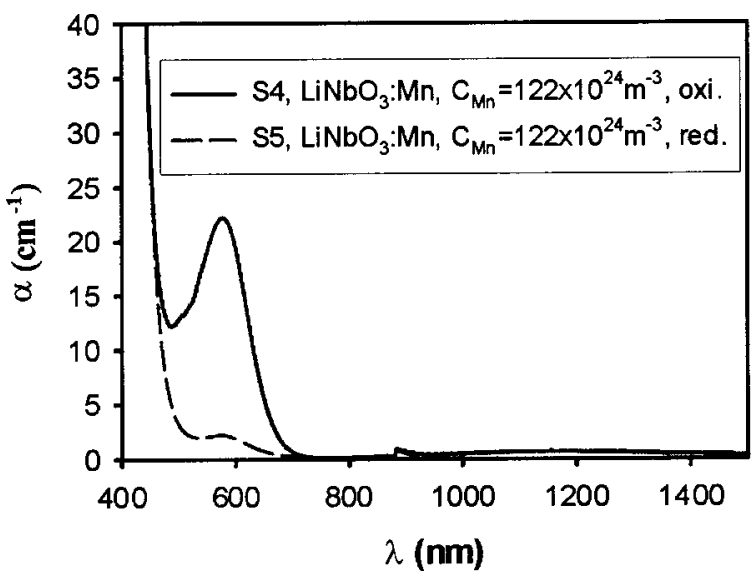

Fig. 3. Absorption coefficient $\alpha$ versus light wavelength $\lambda$ for two $\mathrm{LiNbO}_{3}$ crystals doped with 0.5 wt. $\% \mathrm{MnCO}_{3}$ for ordinary light polarization. One was oxidized, and the other one was reduced.

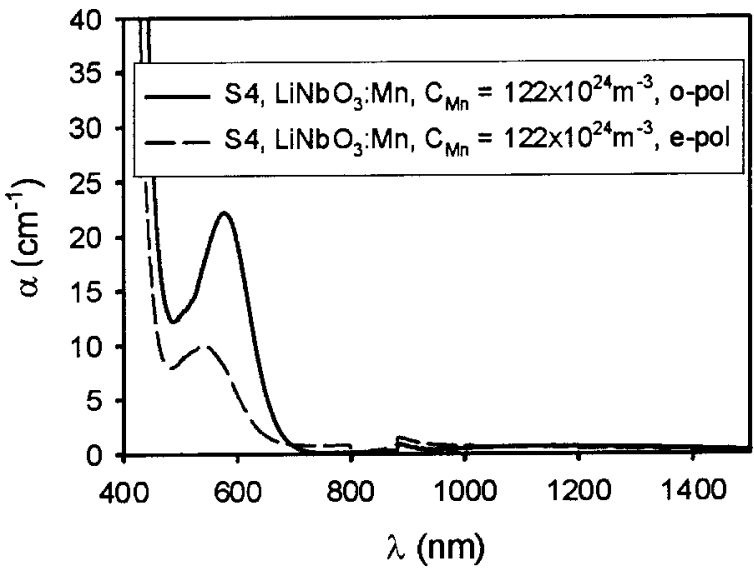

Fig. 4. Absorption coefficient $\alpha$ versus light wavelength $\lambda$ for a $\mathrm{LiNbO}_{3}: \mathrm{Mn}$ crystal for ordinary and extraordinary light polarizations.

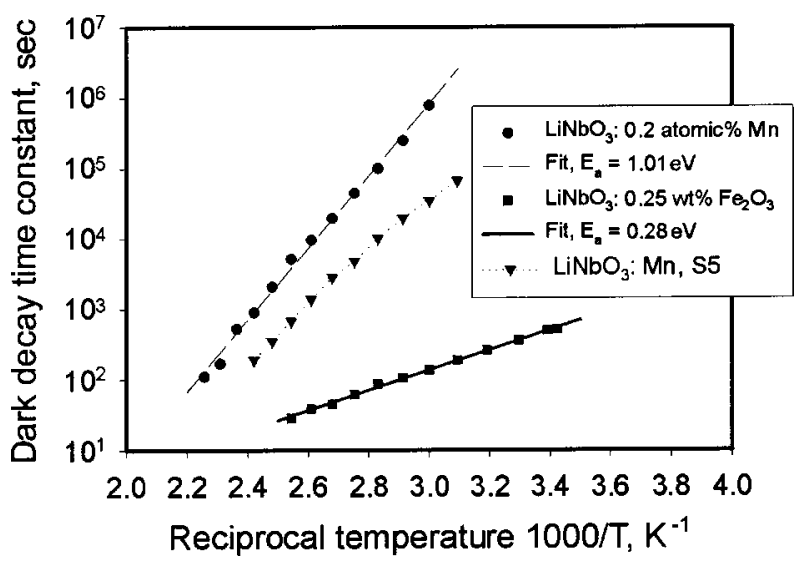

Fig. 5. Measured dark-decay time constant of nonfixed holograms in $\mathrm{LiNbO}_{3}: \mathrm{Mn}$ crystals. For comparison, the measured dark decay of a $\mathrm{LiNbO}_{3}: \mathrm{Fe}$ crystal doped with $0.25 \mathrm{wt} . \% \mathrm{Fe}_{2} \mathrm{O}_{3}$ is included.

with wavelength $514 \mathrm{~nm}$ was used to record holograms. The laser beam was split into two equal-intensity extraordinarily polarized beams that were expanded to cover the whole crystal during recording. Recorded holograms had a grating period of $1.3 \mu \mathrm{m}$ and were written with the grating vector oriented along the $c$ axis. Recording was performed at room temperature. Afterwards, the crystals were heated to a certain temperature in the dark, and a weak laser beam of $514 \mathrm{~nm}$ was used to monitor the holographic diffraction efficiency. The weak readout light illuminated the crystal only from time to time, and the intervals between two measurements were long enough to keep the erasure of the holograms by the probing beam negligible. After each experiment, the crystal was heated to $230{ }^{\circ} \mathrm{C}$ and kept at this temperature under uniform illumination for $\sim 45 \mathrm{~min}$. to erase the gratings completely.

The results are shown in Fig. 5. For comparison, also included in Fig. 5 is the measured dark decay of a $\mathrm{LiNbO}_{3}: \mathrm{Fe}$ crystal doped with 0.25 wt. $\% \mathrm{Fe}_{2} \mathrm{O}_{3}$. We can see that the dark-decay time constants in both, $\mathrm{LiNbO}_{3}: 0.2$ at. $\% \mathrm{Mn}$ and $\mathrm{LiNbO}_{3}: 0.25$ wt. $\% \mathrm{Fe}_{2} \mathrm{O}_{3}$, obey an Arrhenius-type dependence on the absolute temperature $T$, but with two different activation energies, $1.0 \mathrm{eV}$ and $0.28 \mathrm{eV}$, respectively, which indicates two distinct dominant dark-decay mechanisms: proton compensation and electron tunneling. ${ }^{9,10}$ Generally, both of these two mechanisms contribute to the dark decay in $\mathrm{LiNbO}_{3}$ crystals, but in $\mathrm{LiNbO}_{3}$ crystals with low doping levels, electron tunneling is very weak and the dark decay is dominated by proton compensation. With the doping levels increasing, the effect of electron tunneling becomes stronger and stronger. For example, in $\mathrm{LiNbO}_{3}: \mathrm{Fe}$ crystals with doping levels above $0.1 \mathrm{wt} . \% \mathrm{Fe}_{2} \mathrm{O}_{3}$, the effect of electron tunneling becomes the dominant dark-decay origin and limits the highest practical doping level below 0.1 wt. $\% \mathrm{Fe}_{2} \mathrm{O}_{3}$. The possibility of electron tunneling through a square potential is determined by Eq. (1). Since the Mn center is deeper than the Fe center, the height of the barrier of Mn center should be larger than that of the Fe center. The effect of electron tunneling in $\mathrm{LiNbO}_{3}: 0.2$ at. $\% \mathrm{Mn}$ is negligible, and the dark decay is dominated by proton compensation. 
From Fig. 5 we also can see that the single Arrhenius law does not hold for $\mathrm{S} 5$, a $\mathrm{LiNbO}_{3}: \mathrm{Mn}$ crystal doped with 0.5 wt. $\% \mathrm{MnCO}_{3}$, in the temperature range we used here. This means that both proton compensation and electron tunneling contribute to the dark decay in S5. Nevertheless, the effect of electron tunneling in S5 is still very small. If we fit the data obtained from S5 for low temperatures to the Arrhenius law, we get the activation energy of S5 for electron tunneling as $0.52 \mathrm{eV}$. This value is smaller than that of proton compensation, which is $\sim 1.0$ $\mathrm{eV}$, but much larger than that of electron tunneling of $\mathrm{LiNbO}_{3}: \mathrm{Fe}, 0.28 \mathrm{eV}$. The lifetime of nonfixed holograms in S5 at room temperature is $\sim 17$ days. ${ }^{10}$ Although this kind of lifetime is not suitable for some applications such as optical add/drop filters for optical telecommunications, it is acceptable for write/rewrite holographic memories. The measured dark-decay time constants in S5 suggest that the highest practical doping level of $\mathrm{LiNbO}_{3}: \mathrm{Mn}$ is $\sim 0.5$ wt. $\% \mathrm{MnCO}_{3}$.

\section{Dynamic Range and Sensitivity}

Dynamic range and sensitivity are two of the most important system metrics for holographic storage systems, which are relevant for almost all the system parameters. One of the key considerations for holographic storage systems is the selection of the recording wavelength. Typically, the optimal recording wavelength is the center wavelength of the absorption band corresponding to the photorefractive excitation of electrons from donors. We can see from Subsection 4.A that there is no apparent absorption peak due to the photorefractive excitation. Nevertheless, we experimentally found that, in terms of dynamic range and sensitivity, the optimal recording wavelength for $\mathrm{LiNbO}_{3}: \mathrm{Mn}$ crystals is $458 \mathrm{~nm}$ among all the wavelengths of laser sources available in our lab, which include $401 \mathrm{~nm}, 458 \mathrm{~nm}, 477 \mathrm{~nm}, 488 \mathrm{~nm}, 514 \mathrm{~nm}$, and $633 \mathrm{~nm}$. In the following experiments of photorefractive recording, laser beams with wavelength of 458 $\mathrm{nm}$ are used.

Dynamic range or $M / \#$ of a holographic storage system is proportional to the maximum refractive-index change, $\Delta n_{s}$, that can be achieved in the recording material. In

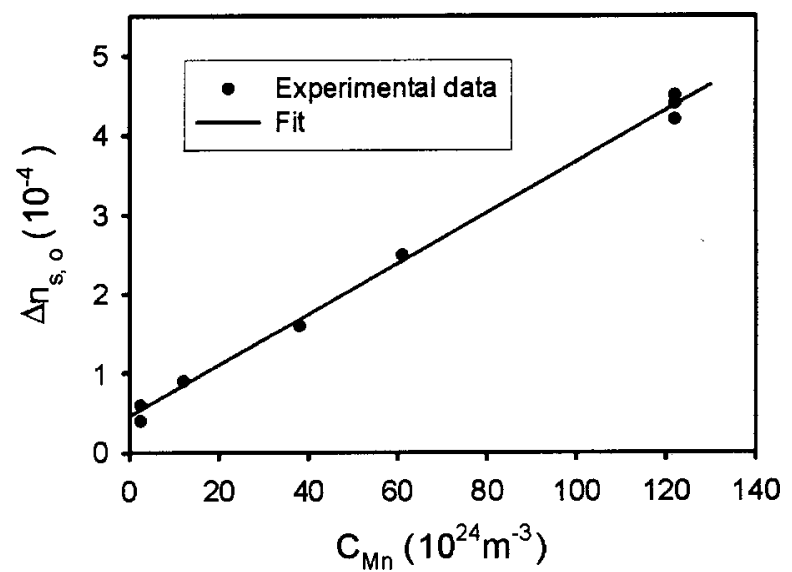

Fig. 6. Measured maximum refractive-index change $\Delta n_{\mathrm{s}, \mathrm{o}}$ for several Mn-doped lithium niobate crystals with different $\mathrm{Mn}$ concentrations $C_{\mathrm{Mn}}$ (wavelength $458 \mathrm{~nm}$, ordinary light polarization). transmission recording geometry with lithium niobate crystals, although the local recording speeds vary inside the crystal over the thickness because of the different recording intensities due to absorption, the saturation value of the refractive-index changes $\Delta n_{s}$ is the same for the whole crystal, if the effect of self-diffraction can be ignored during recording. Therefore if the recording time is long enough and the recording is so stable that we can record the grating everywhere inside the crystal to saturation, we can obtain a uniform volume holographic grating. Then the maximum refractive-index change can be calculated from the measured saturation value of the diffraction efficiency by Eq. (6).

Figure 6 shows the measured maximum refractiveindex change $\Delta n_{s, o}$ of several Mn-doped lithium niobate crystals with different Mn concentrations. All the crystals are in the as-grown state. Ordinary polarization is used. The recording is stabilized utilizing an active feedback system. ${ }^{30}$ From Fig. 6, we can see that a linear dependence of $\Delta n_{s, o}$ on the Mn concentration is still valid even for the highly doped crystal with a Mn concentration of $122 \times 10^{24} \mathrm{~m}^{-3}$. Considering that the highest practical concentration of iron in Fe-doped lithium niobate crystals is $\sim 40 \times 10^{24} \mathrm{~m}^{-3}, 8$ it is obvious that the electrontunneling effect, which is the limiting factor for the highest practical doping level of crystals, is much smaller in Mn-doped crystals than that in Fe-doped crystals. By using $\mathrm{Mn}$ instead of $\mathrm{Fe}$ as the dopant, we can increase the highest practical doping level by a factor of $\sim 3$. From Eq. (1) we can deduce that for $V \gg E$, this yields a ratio of $\left(3^{2}\right)^{1 / 3}=2.1$ between the heights of barriers for $\mathrm{Mn}$ doped and Fe-doped crystals. This is very consistent with the ratio of the thermal-activation energies of dark decay $0.52 / 0.28=1.8$ (Subsection 4.B). Notice that the magnitudes of the largest $\Delta n_{s}$ that can be obtained for Fedoped and Mn-doped crystals are almost the same. ${ }^{8}$ The reason that the maximum refractive-index change in $\mathrm{LiNbO}_{3}: \mathrm{Mn}$ is less than that in $\mathrm{LiNbO}_{3}: \mathrm{Fe}$ with the same doping level is probably because the bulk photovoltaic coefficient of $\mathrm{Mn}$ is smaller than that of $\mathrm{Fe}$ in lithium niobate crystals.

The sensitivity is defined by Eq. (4), that is, the initial recording slope normalized by the thickness of the crystal and the recording intensity. Figure 7 shows the measured sensitivities of the Mn-doped crystals with different Mn concentrations for ordinary light polarization. The crystals are the same as we used for Fig. 6. We can see that the sensitivity of $\mathrm{Mn}$-doped lithium niobate crystals increases linearly with the doping level.

$\Delta n_{s}$ is proportional to $\gamma_{\mathrm{eff}} n_{\mathrm{eff}}^{3}$ [Eq. (5)]. $M / \#$ and sensitivity are proportional to $\Delta n_{s}$, and hence $M / \#$ and sensitivity are proportional to $\gamma_{\mathrm{eff}} n_{\text {eff }}^{3}$. The electro-optic coefficient of $\mathrm{LiNbO}_{3}$ for extraordinary light polarization is 3.2 times as large as that for ordinary light polarization. Then by using extraordinary polarization instead of ordinary polarization, we can boost $M / \#$ and sensitivity of a $\mathrm{LiNbO}_{3}$-based holographic storage system by a factor of $\sim 3$. For $\mathrm{LiNbO}_{3}: \mathrm{Mn}$ crystals, by use of extraordinary polarization, extra gains of $M / \#$ and sensitivity will result from the large dichroism, that is, the smaller absorption coefficient for extraordinary light polarization. For example, with ordinary polarization, the measured $M / \#$ and 


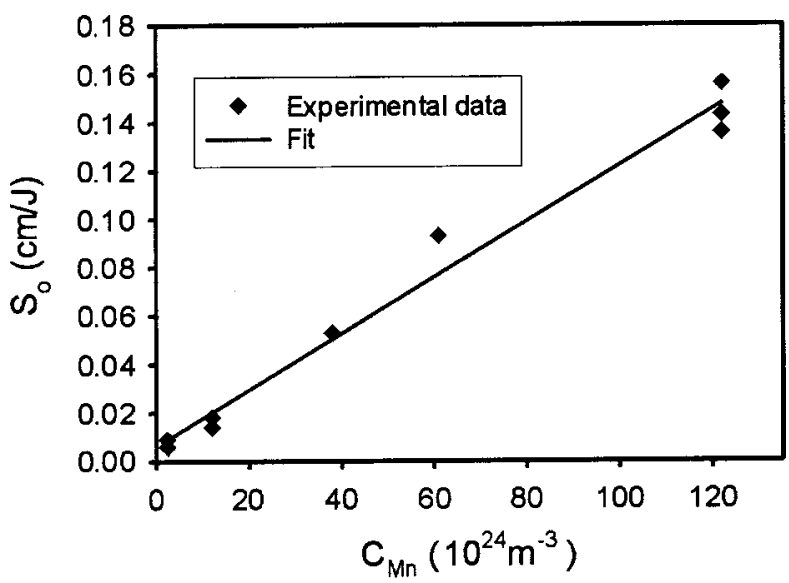

Fig. 7. Measured sensitivities $S_{\text {o }}$ of the Mn-doped crystals with different $\mathrm{Mn}$ concentrations $C_{\mathrm{Mn}}$ for ordinary light polarization (wavelength $458 \mathrm{~nm}$ ).

sensitivity of one of the $\mathrm{LiNbO}_{3}: \mathrm{Mn}$ crystals with a $\mathrm{Mn}$ concentration of $122 \times 10^{24} \mathrm{~m}^{-3}, \mathrm{~S} 4$, are 1.1 and $0.2 \mathrm{~cm} / \mathrm{J}$, respectively, and with extraordinary polarization the measured $M / \#$ and sensitivity of this crystal are 7.3 and $1.3 \mathrm{~cm} / \mathrm{J}$, respectively. Both $M / \#$ and sensitivity gain a factor of $\sim 6.6$ for extraordinary polarization, instead of 3 .

Inside the crystal, the intensity is a function of depth and can be represented as

$$
I(x)=(1-R) I_{0} \exp (-\alpha x / \cos \theta),
$$

where $R$ is the reflectivity, $I_{0}$ is the incident intensity outside the crystal, $\alpha$ is the absorption coefficient, $x$ is the depth, and $\theta$ is the incident angle inside the crystal. The recording time constant $\tau$ is inversely proportional to the recording intensity; therefore the local grating strength during recording can be represented as a function of time and depth as

$$
A(x)=A_{0}[1-\exp (-t / \tau)]=A_{0}\{1-\exp [-c t I(x)]\},
$$

where $c$ is some constant. The initial recording slope can be calculated as

$$
\begin{aligned}
\left.\frac{\mathrm{d} \sqrt{\eta}}{\mathrm{d} t}\right|_{t \ll \tau_{e}} & =\exp \left(\frac{-\alpha L}{2 \cos \theta}\right) \times\left.\frac{\mathrm{d}}{\mathrm{d} t}\left[\int_{0}^{L} A(x) \mathrm{d} x\right]\right|_{t \ll \tau_{e}} \\
& \propto \frac{\exp (-\alpha L / 2 \cos \theta)-\exp (-3 \alpha L / 2 \cos \theta)}{\alpha} .
\end{aligned}
$$

For the crystal for which we measured $M / \#$ and sensitivity using both ordinary and extraordinary polarization, $\mathrm{S} 4$, the thickness $L$ is $0.84 \mathrm{~mm}$, and the absorption coefficients for ordinary and extraordinary polarization are $20.9 \mathrm{~cm}^{-1}$ and $11.4 \mathrm{~cm}^{-1}$, respectively. Calculated from Eq. (13), the factor of gain on $M / \#$ and sensitivity due to the dichroism of this crystal is 2.0. Multiplying this value with the ratio between the factor $\gamma_{\text {eff }} n_{\text {eff }}^{3}$ of extraordinary and ordinary polarizations, 3 , gives us the theoretical factor of gains on $M / \#$ and sensitivity with extraordinary light polarization as 6 , which agrees with the experimental result very well.
Another advantage that we found in manganese-doped lithium niobate crystals for holographic storage is the excellent recording repeatability, even without an active stabilization of the setup. The good repeatability and stability of recording in $\mathrm{LiNbO}_{3}: \mathrm{Mn}$ crystals probably come from the high sensitivity and the low holographic scattering.

One interesting observation for the highly doped $\mathrm{LiNbO}_{3}: \mathrm{Mn}$ crystals with extraordinary light polarization is the oscillating behavior of the recording curves: the diffraction efficiency goes up to some maximum value, then drops down to some minimum value, then it goes up and drops down again. There are two possible origins for such oscillations: One is a complex recording time constant due to a phase shift between intensity pattern and refractive-index change pattern. ${ }^{31}$ The other is the $\sin ^{2}$ behavior of the diffraction efficiency due to the large refractive-index change. In order to sort out the real origin behind the oscillating behavior, a red readout beam was added to monitor the temporal evolution of grating strength. Figure 8 shows one recording and erasure curve using laser light with wavelength of $458 \mathrm{~nm}$ and extraordinary light polarization without active phase stabilization of the setup. Here we modify the definition of diffraction efficiencies as

$$
\eta^{\prime}=\frac{I_{\text {diffracted }}}{I_{\text {diffracted }}+I_{\text {transmitted }}} .
$$

The recording and erasure beams were two plane waves with the wavelength of $458 \mathrm{~nm}$ and extraordinary polarization. The recording intensity was $11.5 \mathrm{~mW} / \mathrm{cm}^{2}$ per beam. During recording and erasure, a red readout beam with the wavelength $633 \mathrm{~nm}$ and ordinary polarization was added to monitor the temporal evolution of the refractive-index changes. Figure 8 explicitly shows that the oscillating behavior of the recording curves is simply caused by the $\sin ^{2}$ behavior of $\eta^{\prime}$ instead of the complex recording time constant. Otherwise, we should see the same oscillation for the recording curve read out by the red beam. In fact, the diffraction efficiency read out by

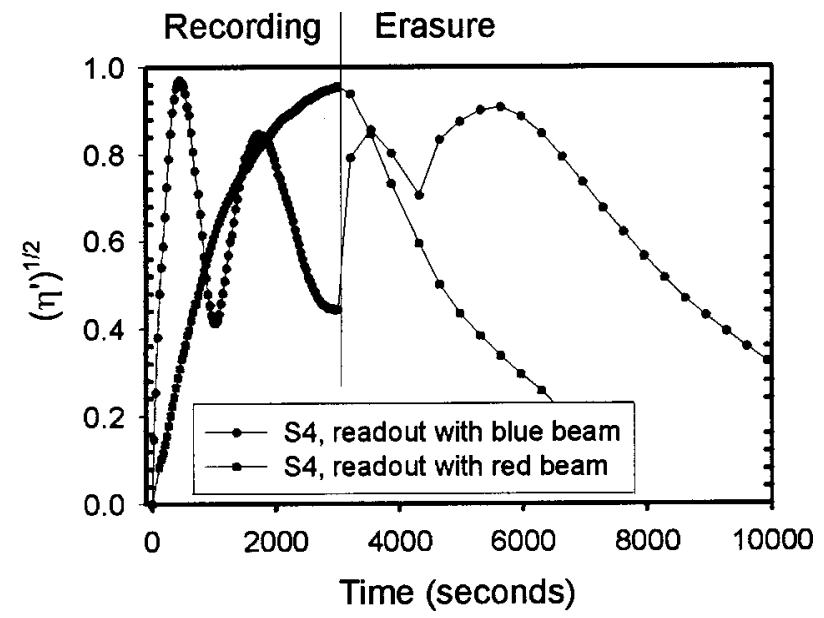

Fig. 8. Recording and erasure curves for sample S4. The recording and erasure beams are two plane waves with the wavelength of $458 \mathrm{~nm}$ and extraordinary polarization. Another red beam with the wavelength of $633 \mathrm{~nm}$ and ordinary polarization was added to monitor the temporal evolution of the gratings. 

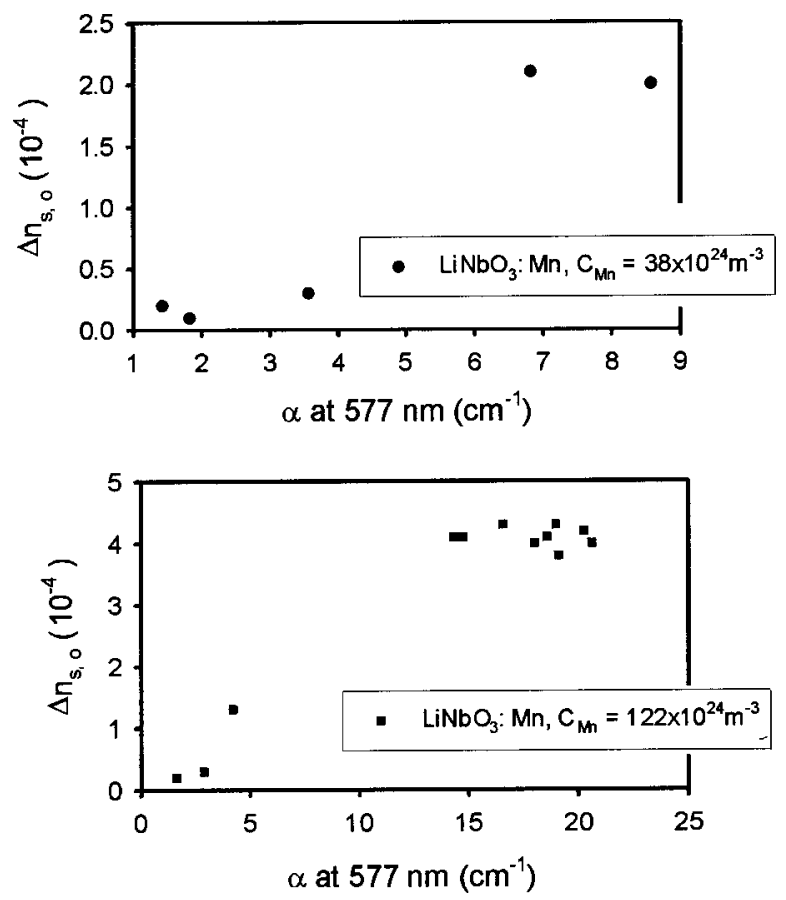

Fig. 9. Measured maximum refractive-index change $\Delta n_{\mathrm{s}, \mathrm{o}}$ for ordinary light polarization in the $\mathrm{LiNbO}_{3}$ crystals doped with 0.2 at.\% $\mathrm{Mn}$ and with $0.5 \mathrm{wt} . \% \mathrm{MnCO}_{3}$ for different oxidation states that are quantified by the absorption coefficient at $577 \mathrm{~nm}$.

the red beam kept growing during the recording even when the diffraction efficiency read out by the blue beam decreased. That the diffraction efficiency of the first maximum read out by the blue beam is close to $100 \%$ also suggests the $\sin ^{2}$ behavior. Using the coupled-wave theory, we can obtain the diffraction efficiency in symmetric transmission geometry, as in Eq. (6). If we use the modified definition of diffraction efficiencies, then we have ${ }^{20}$

$$
\eta^{\prime}=\sin ^{2}\left(\frac{\pi L \Delta n}{\lambda \cos \theta}\right)
$$

The recording curve read out by the blue beam in Fig. 8 is over two maxima and reaches the second minimum at the end of recording. From the recording curve read out by the blue beam, we can estimate the amplitude of the refractive-index change for the blue beam, $\Delta n_{b}$, at the end of recording as $1.2 \times 10^{-3}$.

The oxidation state of $\mathrm{LiNbO}_{3}$ crystals can be changed by annealing at elevated temperature in appropriate atmosphere, typically oxygen for oxidation and argon for reduction. It is well known that $M / \#$ and sensitivity in $\mathrm{LiNbO}_{3}: \mathrm{Fe}$ crystals are strong functions of the oxidation state. Typically, the more the crystal is reduced, the larger the sensitivity and the smaller the $M / \#$, and vice versa. In Ref. 11, we reported the measured $M / \#$ and sensitivity in a $\mathrm{LiNbO}_{3}$ crystal doped with 0.2 at.\% $\mathrm{Mn}$ with different oxidation states. We found that the measured sensitivity in that sample is $0.5 \mathrm{~cm} / \mathrm{J}$ and almost independent of the annealing state, while the $M / \#$ drops by a factor of 15 from the highly oxidized to the highly reduced state. Figure 9 shows the measured maximum refractive-index change for ordinary polarization in the
$\mathrm{LiNbO}_{3}$ crystals doped with 0.2 at.\% $\mathrm{Mn}$ and with 0.5 wt. $\% \mathrm{MnCO}_{3}$ having different oxidation states. We also measured the $M / \#$ and sensitivity of the highly doped $\mathrm{LiNbO}_{3}$ crystal that contains 0.5 wt. $\% \mathrm{MnCO}_{3}$ having different oxidation states using extraordinary light polarization. The thickness of the crystal is $0.84 \mathrm{~mm}$. From the highly reduced to the highly oxidized state, the $M / \#$ increases from 4.5 to 7.3 , while the measured sensitivity increases from $0.8 \mathrm{~cm} / \mathrm{J}$ to $1.3 \mathrm{~cm} / \mathrm{J}$; that is, the more oxidized the crystal, the larger $M / \#$ and sensitivity we obtained. In the crystals with doping levels 0.2 at.\% $\mathrm{Mn}$ or $0.5 \mathrm{wt} . \% \mathrm{MnCO}_{3}$, the optimal oxidation states in terms of both $M / \#$ and sensitivity are highly oxidized. The lack of the trade-off between $M / \#$ and sensitivity is good for, e.g., holographic storage systems.

In the one-center model for $\mathrm{LiNbO}_{3}: \mathrm{Mn}, \mathrm{Mn}^{2+}$ sites act as filled traps and electrons can be optically excited from $\mathrm{Mn}^{2+}$ sites to the conduction band. $\mathrm{Mn}^{3+}$ sites act as empty traps with which electrons in the conduction band can recombine. In all experiments, we use transmission geometry, for which the grating vector $K$ is small, and we would expect that

$$
E_{\mathrm{q}} \gg E_{\mathrm{ph}} \gg E_{\mathrm{d}} .
$$

Under these conditions, combining Eqs. (3)-(9) and

$$
\tau_{e} \propto \frac{\epsilon}{q \mu s I} \frac{r_{A} N_{A}}{\left(N_{D}-N_{A}\right)},
$$

we obtain

$$
\begin{aligned}
M / \# \propto E_{\mathrm{sc}} & \approx E_{\mathrm{ph}} \equiv \frac{p r_{A} N_{A}}{q \mu s} \propto N_{A}, \\
S & \propto \frac{M / \#}{I \tau_{e}} \propto \frac{E_{\mathrm{ph}}}{I \tau_{e}}=\frac{p\left(N_{D}-N_{A}\right)}{\epsilon} \propto N_{D}-N_{A} .
\end{aligned}
$$

One way to check the validity of conditions in Eq. (16) is to record holograms with different grating vector or period length. The grating period in transmission geometry can be varied by changing the outside angle between the recording beams. Since the bulk photovoltaic field $E_{\mathrm{ph}}$ is independent of the grating period [Eq. (9)], if the conditions in Eq. (16) are valid, then the recording curves with different grating periods should not be different. Figure 10 shows two recording curves in sample S4 with different grating periods. We can see that those two recording curves are almost overlapped. This means that for $\mathrm{LiNbO}_{3}: \mathrm{Mn}$ crystals in transmission geometry, it is the photovoltaic current that dominates the photorefractive effect, and the $M / \#$ is proportional to the photovoltaic field $E_{\mathrm{ph}}$.

\section{Holographic Scattering}

Holographic scattering or fanning in lithium niobate can build up during recording or readout as parasitic holograms. In general, fanning is a serious problem for holographic storage systems with photorefractive lithium niobate crystals because it is unpredictable, and if allowed to build up for a long time, eventually it leads to complete deterioration of the performance. It is well known that this is the reason that many of the large-scale demonstrations of holographic memories were done in the 90-deg. 
geometry where fanning is less of a problem. ${ }^{6}$ In the previous subsection, we have already shown that we can obtain larger $M / \#$ and sensitivity in $\mathrm{LiNbO}_{3}: \mathrm{Mn}$ by using transmission geometry and extraordinary polarization. It is also known that $\mathrm{LiNbO}_{3}: \mathrm{Fe}$ crystals with high sensitivity is prone to fanning. Considering the high sensitivity that we measured in $\mathrm{LiNbO}_{3}: \mathrm{Mn}$ crystals, one may argue that fanning is a serious problem. Nevertheless, experimentally, we observed little holographic scattering of $\mathrm{LiNbO}_{3}: \mathrm{Mn}$ crystals during the recording and readout.

We performed an experiment to quantitatively assess the fanning behavior of several $\mathrm{LiNbO}_{3}: \mathrm{Mn}$ and $\mathrm{LiNbO}_{3}: \mathrm{Fe}$ crystals that had approximately the same thickness. The optical setup is shown in Fig. 11. One plane wave with extraordinary polarization was split by a beam splitter into two beams, one of which was directed to the crystal with normal incidence, the other was directed to a detector and as the reference beam. The power of the transmitted beam after the crystal was measured from time to time. We used the laser beams with wavelengths of $458 \mathrm{~nm}$ for $\mathrm{LiNbO}_{3}: \mathrm{Mn}$ and $488 \mathrm{~nm}$ for

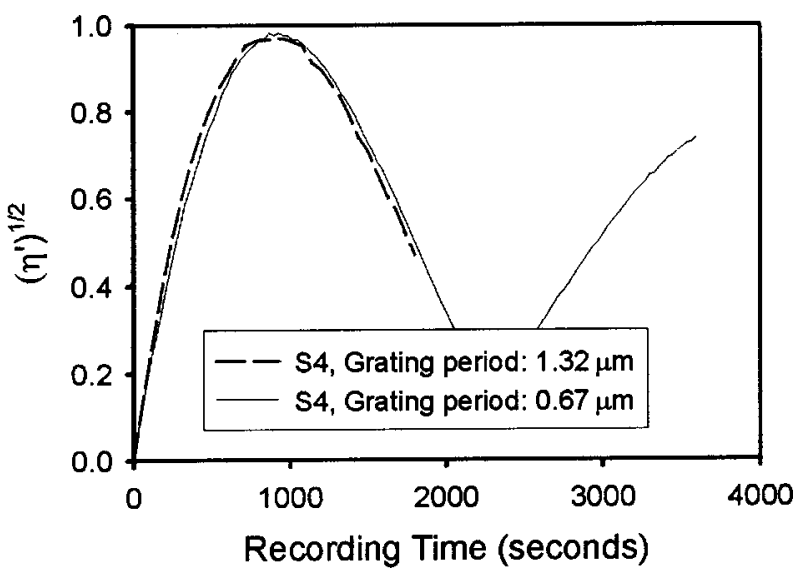

Fig. 10. Diffraction efficiency versus time for recording of two gratings under identical conditions, except that the period length of the grating differs by a factor of 2 (wavelength $458 \mathrm{~nm}$, extraordinary light polarization).

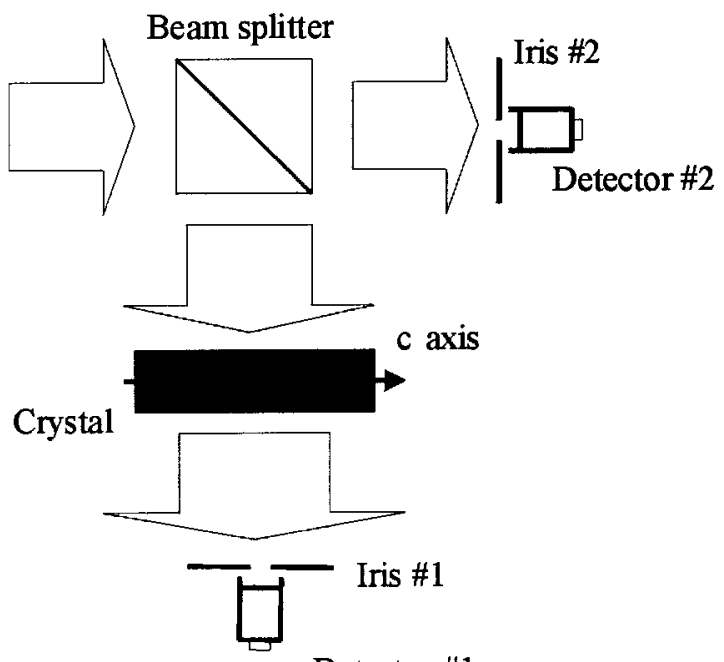

Detector \#1

Fig. 11. Optical setup for the measurements of fanning in lithium niobate crystals.

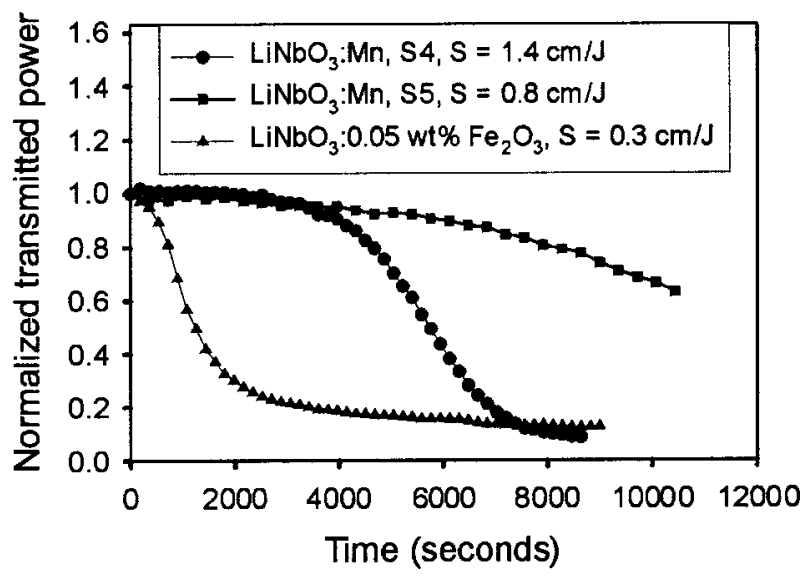

Fig. 12. Light power that is transmitted through the crystal during the fanning measurements.

$\mathrm{LiNbO}_{3}: \mathrm{Fe}$, and the average intensities in all the cases were the same, $7 \mathrm{~mW} / \mathrm{cm}^{2}$. In Fig. 12 we plot the normalized transmitted power as a function of time. Clearly, from the very beginning, the holographic scattering in $\mathrm{LiNbO}_{3}: \mathrm{Fe}$ built up very quickly, whereas $\mathrm{LiNbO}_{3}: \mathrm{Mn}$ crystals remain almost unaffected after one hour. Note that the sensitivities of $\mathrm{LiNbO}_{3}: \mathrm{Mn}$ crystals we used in this experiment are much higher than those of the $\mathrm{LiNbO}_{3}: \mathrm{Fe}$ crystal, and the buildup speeds of fanning in $\mathrm{LiNbO}_{3}: \mathrm{Mn}$ crystals are much slower. In fact, with this intensity, $7 \mathrm{~mW} / \mathrm{cm}^{2}$, we can record holograms to almost saturation in S4 within an hour, just before fanning could build up. From these measurements we see that, indeed, fanning is dramatically reduced in $\mathrm{LiNbO}_{3}: \mathrm{Mn}$ crystals for recording with blue light.

\section{E. Thermal Fixing in Mn-Doped Lithium Niobate Crystals}

Stabler et al. found that holograms can be thermally fixed in some electro-optic materials, such as $\mathrm{LiNbO}_{3}$ crystals, by heating the crystals during or after normal recording. ${ }^{32}$ The fixed holograms can then be read out nondestructively at room temperature. It has been shown that protons play a key role in thermal fixing. ${ }^{33-37}$ The mobility of protons obeys the so-called Arrhenius law with an activation energy of $1.0-1.2 \mathrm{eV}$ in the temperature range $20-300{ }^{\circ} \mathrm{C} .{ }^{32,38}$ From room temperature to enhanced temperature, the mobility of protons increases exponentially. At enhanced temperatures, the electric space-charge field gratings are compensated by protonic gratings because protons become mobile. After cooling, the crystal down to room-temperature homogeneous illumination is used to develop the hologram. Homogeneous illumination yields spatially modulated currents because of the grating of photoexcitable electrons that is fixed due to the protonic grating. ${ }^{34}$ The spatially modulated currents build up space-charge fields and refractive-index changes. In other words, due to the development light, the phase shift between the electronic and the ionic gratings is changed in such a way that the initial full compensation of these two gratings is partly released. The mobility of protons at room temperature is very small, which leaves the protonic gratings at room temperature nonde- 


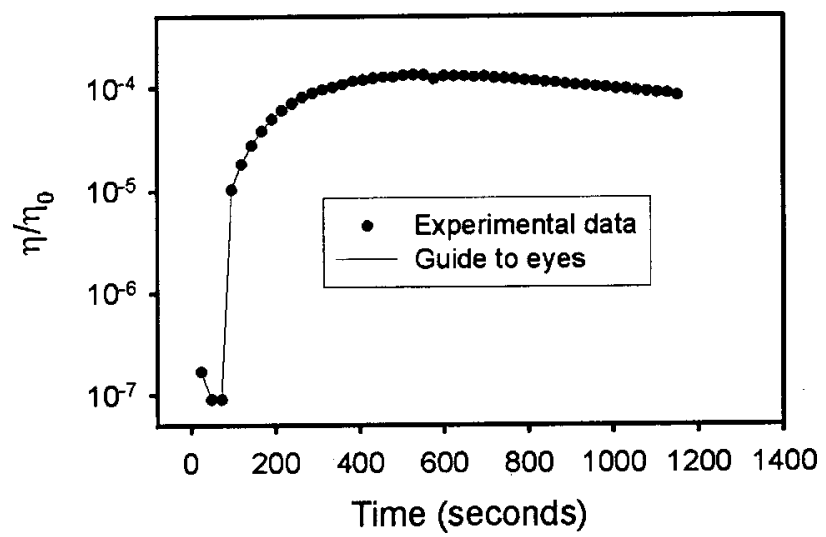

Fig. 13. Diffraction efficiency versus time for development of a thermally fixed hologram.

structive to the illumination of readout beam. Depending on whether the recording is performed at room temperature or enhanced temperature, we can denote the schedule of thermal fixing as "low-high-low" or "high-low".

For the low-high-low process, the most important issue is the thermal-fixing efficiency, which is defined as the ratio of diffraction efficiency after and before thermal fixing. Up to more than 90\% thermal-fixing efficiencies have been reported in weakly doped $\mathrm{LiNbO}_{3}: \mathrm{Fe}$. We successfully performed the low-high-low thermal fixing in $\mathrm{LiNbO}_{3}: \mathrm{Mn}$ crystals. Figure 13 shows the thermal fixing in one of the manganese-doped lithium niobate crystals with doping level 0.2 wt.\% $\mathrm{MnO}, \mathrm{S} 1$. The vertical axis is the normalized diffraction efficiency with the diffraction efficiency just after usual recording normalized to 1 . Recording was performed at room temperature by two plane waves with the wavelength $458 \mathrm{~nm}$ and extraordinary polarization. After recording, a red beam was used to scan over some angle range that covers the Bragg-matched position to find the maximum diffraction efficiency. Then the crystal was put into an oven with temperature preset to $160{ }^{\circ} \mathrm{C}$. Afterwards, the crystal was taken out of the oven and cooled down to room temperature in the air. Homogeneous illumination with blue light was used to develop the fixed hologram off-Bragg. Simultaneously, a red readout beam was used to scan some angle range to find the Bragg-matched diffraction efficiency.

From Fig. 13 we can see that, at the beginning of revealing, the diffraction efficiency is very low. The electronic grating was compensated by the protonic grating. The reason we see the decrease of the diffraction efficiency at the beginning of the development is that the compensation was not completed. The final small thermal-fixing efficiency $\left(\sim 10^{-4}\right)$ indicates that the effective modulation depth of the fixed electronic pattern is $10^{-4}$. This low thermal-fixing efficiency is also observed for $\mathrm{LiNbO}_{3}: \mathrm{Mn}$ crystals with other doping levels. We got thermal-fixing efficiencies of $8 \times 10^{-4}$ and $5.2 \times 10^{-4}$ for $\mathrm{LiNbO}_{3}$ samples doped with 0.05 wt. $\% \mathrm{MnO}$ and with 0.1 wt.\% $\mathrm{MnO}$, respectively. The small "low-high-low" thermal-fixing efficiency is one drawback of manganesedoped lithium niobate crystals.

\section{DISCUSSION}

In the previous section, we have experimentally shown that $\mathrm{LiNbO}_{3}: \mathrm{Mn}$ crystals are highly suited for holo- graphic storage. In order to tailor the material properties for holographic applications, a quantitative description of the photorefractive properties is needed. First, we will discuss the dominant charge driving force. Then we will concentrate on a charge-transport model for the crystals that are doped with up to 0.2 wt.\% Mn. Finally, we will discuss the behavior of crystals with higher doping levels.

\section{A. Dominant Charge Driving Force}

For the case that the bulk photovoltaic effect is the dominant charge driving force, $M / \#$, sensitivity, and also the temporal evolution of refractive-index changes and diffraction efficiencies should not depend on the period length of the grating. As Fig. 10 shows, this is indeed the case. Furthermore, we have not seen any experimental evidence for space-charge limiting effects. E.g., the curves in Fig. 10 are identical because of lack of spacecharge limitation. Thus as we have already written it in Eq. (18), the saturation space-charge field $E_{\mathrm{sc}}$ should be equal to $E_{\mathrm{ph}}$.

\section{B. Charge-Transport Model}

Because the samples are doped with $\mathrm{Mn}$, it is evident that $\mathrm{Mn}$ will be the dominant photorefractive center. It is known that $\mathrm{Mn}$ occurs in $\mathrm{LiNbO}_{3}$ in the valence states $2+$ and $3+.^{12-15}$ As we already pointed out, the results can be explained within a $\mathrm{Mn}^{2+/ 3+}$ one-center chargetransport model, assuming that the Mn centers are almost completely filled with electrons, i.e., $c_{\mathrm{Mn}^{2+}} \gg c_{\mathrm{Mn}^{3+}}{ }^{11}$ This explains that on the one hand the $M / \#$ increases, and on the other hand the sensitivity stays constant if one oxidizes the crystals [see Eqs. (18) and (19)], because the $M / \#$ is proportional to $c_{\mathrm{Mn}^{3+}}$ while the sensitivity is proportional to $c_{\mathrm{Mn}^{2+}}=c_{\mathrm{Mn}}-c_{\mathrm{Mn}^{3+}}$. During oxidization $c_{\mathrm{Mn}^{3}}$ increases, but the much larger $c_{\mathrm{Mn}^{2}}$ stays almost constant.

We are now able to perform two quantitative tests of this model: Eq. (9) yields $N_{A}=q \mu s E_{\mathrm{ph}} /\left(p r_{A}\right)$. We will do this for the oxidized crystal that contains 38 $\times 10^{24} \mathrm{~m}^{-3} \mathrm{Mn}$ (see Fig. 9). Here we get a $\Delta n_{s, o}$ of 2.0 $\times 10^{-4}$. With Eq. (5) and with $\gamma_{\text {eff }}=12 \mathrm{pm} / \mathrm{V}$ and $n_{\text {eff }}$ $=2.35$, we get $E_{\mathrm{sc}}=2.6 \times 10^{6} \mathrm{~V} / \mathrm{m}$. All other parameters of the above-mentioned equation to calculate $N_{A}$ are available from literature. ${ }^{18}$ However, in the referenced paper, the parameters $s$ and $p$ were determined for light of the wavelength $366 \mathrm{~nm}$. Anyhow, other authors showed before that the ratio $s / p$ is reduced by only a factor of 2 if one moves from $366 \mathrm{~nm}$ to $458 \mathrm{~nm}$. ${ }^{15}$ Thus we end up with $q=1.6 \times 10^{-19} \mathrm{As}, \mu=7.4 \times 10^{-5} \mathrm{~m}^{2}$ / $(\mathrm{Vs}), \quad s / p=1.6 \times 10^{27} \mathrm{~V} /(\mathrm{Jm}), \quad$ and $\quad r_{A}=2.4 \times 10^{-13}$ $\times \mathrm{m}^{3} / \mathrm{s}$. Plugging in the numbers yields $c_{\mathrm{Mn}^{3+}}=N_{A}$ $=0.2 \times 10^{24} \mathrm{~m}^{-3}$. This is much less than $c_{\mathrm{Mn}}=38$ $\times 10^{24} \mathrm{~m}^{-3}$, verifying the statement $c_{\mathrm{Mn}^{3+}} \ll c_{\mathrm{Mn}}$. Anyhow, $c_{\mathrm{Mn}^{3}}$ is still large enough to avoid space-charge limiting effects. Calculation of $E_{q}$ yields $20 \times 10^{6} \mathrm{~V} / \mathrm{m}$ for a $1-\mu \mathrm{m}$ period length of the grating.

The second test is the calculation of the absorption cross section at $458 \mathrm{~nm}$. The crystal that is doped with $122 \times 10^{24} \mathrm{~m}^{-3} \mathrm{Mn}\left(0.5 \mathrm{wt} . \% \mathrm{MnCO}_{3}\right)$ has at $458 \mathrm{~nm}$ an absorption of $\alpha=2000 \mathrm{~m}^{-1}$. Considering that $\alpha$ $=s_{\mathrm{abs}} c_{\mathrm{Mn}^{2+}}$, we get for $c_{\mathrm{Mn}^{2+}} \approx c_{\mathrm{Mn}}$ the result $s_{\mathrm{abs}}$ 
$=1.64 \times 10^{-23} \mathrm{~m}^{2}$. The photon-absorption cross sections $s_{\text {abs }}$ and $s$ are related through $s=Q s_{\text {abs }}$, where $Q$ is the quantum efficiency for excitation of an electron upon absorption of a photon. A typical value for this $Q$ is $0.05 .^{39,40}$ Thus we end up with $s=8.2 \times 10^{-25} \mathrm{~m}^{2}$. From spectroscopic investigations it is furthermore known that, moving from $458 \mathrm{~nm}$ to $366 \mathrm{~nm}$, the absorption cross section increases roughly by a factor of $10 .^{15}$ This yields $s=8.2 \times 10^{-24} \mathrm{~m}^{2}$. From an independent investigation, the value for $s$ for this wavelength is known; ${ }^{15} s$ was determined to be $1.9 \times 10^{-23} \mathrm{~m}^{2}$. Considering all uncertainties, this agreement is very good and indicates that the assumption $c_{\mathrm{Mn}^{2+}} \approx c_{\mathrm{Mn}}$ is valid.

The concentration ratio $c_{\mathrm{Mn}^{2+}} / c_{\mathrm{Mn}^{3+}}$ should, in analogy to $\mathrm{LiNbO}_{3}: \mathrm{Fe}$, not depend much on the overall doping level for as-grown crystals. Thus it is clear that both $\Delta n_{\mathrm{s}, 0} \propto c_{\mathrm{Mn}^{3+}}$ and $S \propto c_{\mathrm{Mn}^{2+}}$ should grow linearly with the overall $\mathrm{Mn}$ concentration $c_{\mathrm{Mn}}$. This is nicely confirmed by the data shown in Figs. 6 and 7 .

\section{Calibration of the Concentrations}

The insights presented above allow now us to quantify the $\mathrm{Mn}^{2+}$ and the $\mathrm{Mn}^{3+}$ concentrations if the following information is available: Mn concentration in the melt, and absorption coefficient $\alpha_{577 \mathrm{~nm}, 0}$ for ordinary light polarization at $577 \mathrm{~nm}$.

The Mn concentration $c_{\mathrm{Mn}}$ in the crystal is identical to the $\mathrm{Mn}$ concentration in the melt, because we have proved that the distribution coefficient is one. The $\mathrm{Mn}^{2+}$ concentration is even after strong oxidization still in very good approximation given by $c_{\mathrm{Mn}^{2+}}=c_{\mathrm{Mn}}$. And the $\mathrm{Mn}^{3+}$ concentration can be deduced from the absorption band that is due to the crystal field transition. In the abovementioned example, we calculated $c_{\mathrm{Mn}^{3+}}=0.2$ $\times 10^{24} \mathrm{~m}^{-3}$. This crystal has an $\alpha_{577 \mathrm{~nm}, \mathrm{o}}$ of $s=2220$ $\mathrm{m}^{-1}$, thus yielding the relation $c_{\mathrm{Mn}^{3+}}=1.11 \times 10^{20} \mathrm{~m}^{-2}$ $\times \alpha_{577 \mathrm{~nm}, \mathrm{o}}$.

\section{Tailoring of Crystal Properties}

The two key properties are dynamic range and sensitivity. However, there are trade-offs with other properties such as absorption and dark decay. In general, on the one hand, the doping level should be large enough and the crystal should be oxidized enough to satisfy the dynamic range and sensitivity requirements. But on the other hand, it should not be doped or oxidized more than necessary to avoid absorption and accelerated dark decay. As a consequence, for particular applications that require special dynamic range and sensitivity, one needs to know which concentration and annealing state must be selected. With the experimental data provided in this paper and with the concentration calibrations presented above, this is possible:

The $M / \#$ is given by $M / \#=4.5 \times 10^{-22} \mathrm{~m}^{-3} \times c_{\mathrm{Mn}^{3}+}$ per cm, and the sensitivity is $S=1.25 \times 10^{-26} \mathrm{~m}^{-3}$ $\times c_{\mathrm{Mn}^{2+}}$ in $\mathrm{cm} / \mathrm{J}$ for wavelength $458 \mathrm{~nm}$ and extraordinary light polarization.

\section{E. Behavior of Highly Doped Crystals}

All experimental data that are obtained with the crystals that are doped with up to $0.2 \mathrm{wt} . \% \mathrm{MnO}\left(78 \times 10^{24} \mathrm{~m}^{-3}\right)$ can be explained and understood within the one-center $\mathrm{Mn}^{2+/ 3+}$ charge-transport model. However, for crystals with doping levels of 0.5 wt. $\% \mathrm{MnCO}_{3}\left(122 \times 10^{24} \mathrm{~m}^{-3}\right)$, deviations are observed. E.g., the sensitivity increases from 0.8 to $1.3 \mathrm{~cm} / \mathrm{J}$ for oxidation of the crystal, although we would expect that the sensitivity stays constant. So far, we can only speculate where this comes from. The concentration where the deviation from the one-center behavior starts is the same where the tunneling dark-decay mechanism becomes to be present. This may affect the results. Furthermore, for high doping levels, it is possible that Mn occupies different lattice sites or that Mn occurs in more than two valence states. Only additional measurements, e.g., with electron paramagnetic resonance, can clarify the situation in these crystals.

\section{CONCLUSIONS}

In conclusion, we have shown that $\mathrm{LiNbO}_{3}: \mathrm{Mn}$ crystals are very promising for holographic recording. The distribution coefficient of $\mathrm{Mn}$-doped lithium niobate crystals has been determined to be around 1. The effect of dark decay due to electron tunneling, which is the limiting factor for the highest practical doping level, is in $\mathrm{LiNbO}_{3}: \mathrm{Mn}$ less than in $\mathrm{LiNbO}_{3}: \mathrm{Fe}$, and higher doping levels can be used in $\mathrm{LiNbO}_{3}: \mathrm{Mn}$ to achieve larger dynamic range and sensitivity for holographic applications. The highest practical doping level in $\mathrm{LiNbO}_{3}: \mathrm{Mn}$ has been found to be $\sim 0.5$ wt. $\% \mathrm{MnCO}_{3}$, and refractive-index changes and sensitivities up to $1.5 \times 10^{-3}$ and $1.3 \mathrm{~cm} / \mathrm{J}$ are measured for extraordinarily polarized light of the wavelength $458 \mathrm{~nm}$. It has been found that in terms of both dynamic range (or refractive-index change) and sensitivity, the optimal oxidation state is highly oxidized. The material is highly suited for holographic recording by blue light with extraordinary polarization because of a fast response and low absorption. Furthermore, the hologram quality is outstanding because holographic scattering is much weaker compared with that in, e.g., iron-doped lithium niobate. Thermal fixing has been successfully demonstrated in $\mathrm{LiNbO}_{3}: \mathrm{Mn}$ crystals.

\section{ACKNOWLEDGMENTS}

Effort sponsored by the National Science Foundation, the Center for Neuromorphic Systems Engineering, Engineering Research Center, the Defense Advanced Research Projects Agency, and the Deutsche Forschungsgemeinschaft. The authors thank the National Science Foundation and the Deutscher Akademischer Austauschdienst for sponsoring the US-German collaboration, and R. Niedergesäss, GKSS-R. Research Center, Institute for Coastal Research/Physical and Chemical Analysis for the measurements of neutron activation analysis. We thank Ali Adibi for fruitful discussions.

\section{REFERENCES}

1. H. J. Coufal, D. Psaltis, and G. T. Sincerbox, Holographic Data Storage (Springer, New York, 2000).

2. C. Denz, G. Pauliat, G. Roosen, and T. Tschudi, "Volume hologram multiplexing using a deterministic phase encoding method," Opt. Commun. 85, 171-176 (1991). 
3. G. Rakujic, V. Levya, and A. Yariv, "Optical data storage by using wavelength multiplexed volume holograms," Opt. Lett. 17, 1471-1473 (1992).

4. K. Curtis, A. Pu, and D. Psaltis, "Method for holographic storage using peristrophic multiplexing," Opt. Lett. 19, 993-994 (1994).

5. D. Psaltis, M. Levene, A. Pu, G. Barbastathis, and K. Curtis, "Holographic storage using shift multiplexing," Opt. Lett. 20, 782-784 (1995).

6. G. Burr, F. Mok, and D. Psaltis, "Angle and space multiplexed holographic storage using the 90-degree geometry," Opt. Commun. 117, 49-55 (1995)

7. F. Mok, G. Burr, and D. Psaltis, "System metric for holographic memory systems," Opt. Lett. 21, 896-898 (1996).

8. K. Peithmann, A. Wiebrock, and K. Buse, "Photorefractive properties of highly-doped lithium niobate crystals in the visible and near-infrared," Appl. Phys. B 68, 777-784 (1999).

9. I. Nee, M. Müller, K. Buse, and E. Krätzig, "Role of iron in lithium niobate crystals for the dark storage time of holograms," J. Appl. Phys. 88, 4282-4286 (2000).

10. Y. Yang, I. Nee, K. Buse, and D. Psaltis, "Ionic and electronic dark decay of holograms in $\mathrm{LiNbO}_{3}: \mathrm{Fe}$ crystals," Appl. Phys. Lett. 78, 4076-4078 (2001).

11. Y. Yang, K. Buse, and D. Psaltis, "Photorefractive recording in $\mathrm{LiNbO}_{3}$ : Mn," Opt. Lett. 27, 158-160 (2002).

12. O. Thiemann and O. F. Schirmer, "Energy levels of several $3 \mathrm{~d}$ impurities and EPR of $\mathrm{Ti}^{3+}$ in $\mathrm{LiNbO}_{3}$," Proc. SPIE 1018, 18-22 (1988)

13. B. Dischler, J. R. Herrington, and A. Räuber, "Correlation of the photorefractive sensitivity in doped $\mathrm{LiNbO}_{3}$ with chemically induced changes in the optical absorption spectra," Solid State Commun. 14, 1233-1236 (1974).

14. N. N. Sorota and V. P. Yarunichev, "Absorption spectrum of lithium niobate with transition metal impurities in the region 0.3-2.0 $\mu \mathrm{m}$," J. Appl. Spectrosc. 25, 1259-1261 (1976).

15. E. Krätzig and H. Kurz, "Photo-induced currents and voltages in $\mathrm{LiNbO}_{3}$," Ferroelectrics 13, 295-296 (1976).

16. L. Arizmendi, J. M. Cabrera, and F. Agullo-Lopez, "Defects induced in pure and doped $\mathrm{LiNbO}_{3}$ by irradiation and thermal reduction," J. Phys. C: Solid State Phys. 17, 515-529 (1984).

17. K. Buse, A. Adibi, and D. Psaltis, "Non-volatile holographic storage in doubly doped lithium niobate crystals," Nature (London) 393, 665-668 (1998)

18. A. Adibi, K. Buse, and D. Psaltis, "Two-center holographic recording," J. Opt. Soc. Am. B 18, 584-601 (2001).

19. P. Yeh, Introduction to Photorefractive Nonlinear Optics (Wiley, New York, 1993).

20. H. Kogelnik, "Coupled wave theory for thick hologram gratings,” Bell Syst. Tech. J. 48, 2909-2945 (1969).

21. N. V. Kukhtarev, V. B. Markov, S. G. Odoulov, M. S. Soskin, and V. L. Vinetskii, "Holographic storage in electrooptic crystals. I. Steady state," Ferroelectrics 22, 949-960 (1979).

22. E. Krätzig, "Photorefractive effects and photoconductivity in $\mathrm{LiNbO}_{3}$ : Fe," Ferroelectrics 21, 635-636 (1978).

23. E. Krätzig and R. Orlowski, "Light-induced charge transport in doped $\mathrm{LiNbO}_{3}$ and $\mathrm{LiTaO}_{3}$," Ferroelectrics 27, 241 244 (1980).

24. K. Buse, "Light-induced charge transport processes in photorefractive crystals. I. Models and experimental meth- ods,” Appl. Phys. B: Photophys. Laser Chem. 64, 273-291 (1997).

25. K. Buse, "Light-induced charge transport processes in photorefractive crystals. II. Materials," Appl. Phys. B 64, 391-407 (1997).

26. R. Verma, J. Arunachalam, S. Gangadharan, J. C. Vyas, G. P. Kothiyal, and M. K. Gupta, "Trace characterization of lithium niobate by neutron activation analysis," Fresenius J. Anal. Chem. 344, 261-264 (1992).

27. G. Anil, M. Ramanaji, M. R. P. Reddy, A. Kumar, T. L. Prakash, and V. C. Sethi, "ICP-OES determination of Ta, $\mathrm{Nb}, \mathrm{Fe}, \mathrm{Ti}, \mathrm{Sn}, \mathrm{Mn}$, and $\mathrm{W}$ in Indian tantalite-niobate ore concentration using microwave digestion," At. Spectrosc. 23, 32-35 (2002).

28. R. G. Smith, D. B. Fraser, R. T. Denton, and T. C. Rich, "Correlation of reduction in optically induced refractive index inhomogeneity with $\mathrm{OH}$ content in $\mathrm{LiTaO}_{3}$ and $\mathrm{LiNbO}_{3}$," J. Appl. Phys. 39, 4600-4602 (1968).

29. H. J. Reyher, R. Schulz, and O. Thiemann, "Investigation of the optical absorption bands of $\mathrm{Nb}^{4+}$ and $\mathrm{Ti}^{3+}$ in lithium niobate using magnetic circular-dichroism and optically detected magnetic resonance techniques," Phys. Rev. B 50, 3609-3619 (1994).

30. S. Breer, K. Buse, K. Peithmann, H. Vogt, and E. Krätzig, "Stabilized recording and thermal fixing of holograms in photorefractive lithium niobate crystals," Rev. Sci. Instrum. 69, 1591-1594 (1998).

31. N. V. Kukhtarev, "Kinetics of hologram recording and erasure in electrooptic crystals," Sov. Tech. Phys. Lett. 2, 438440 (1976).

32. J. J. Amodei and D. L. Staebler, "Holographic pattern fixing in electro-optic crystals," Appl. Phys. Lett. 18, 540-542 (1971).

33. H. Vormann, G. Weber, S. Kapphan, and E. Krätzig, "Hydrogen as origin of thermal fixing in $\mathrm{LiNbO}_{3}: \mathrm{Fe}$," Solid State Commun. 40, 543-545 (1981).

34. K. Buse, S. Breer, K. Peithmann, S. Kapphua, H. Gao, and E. Krätzig, "Origin of thermal fixing in photorefractive lithium niobate crystals," Phys. Rev. B 56, 1225-1235 (1997).

35. L. Arizmendi, E. M. de Miguel-Sanz, and M. Carrascosa, "Lifetime of thermally fixed holograms in $\mathrm{LiNbO}_{3}: \mathrm{Fe}$ crystals," Opt. Lett. 23, 960-963 (1998).

36. E. M. de Miguel-Sanz, J. Limeres, M. Carrascosa, and L. Arizmendi, "Study of developing of thermally fixed holograms in lithium niobate," J. Opt. Soc. Am. B 17, 1140-1146 (2000)

37. E. M. de Miguel-Sanz, M. Carrascosa, and L. Arizmendi, "Effect of the oxidation state and hydrogen concentration on the lifetime of thermally fixed holograms in $\mathrm{LiNbO}_{3}: \mathrm{Fe}$," Phys. Rev. B 65, 165101 (2002)

38. A. Yariv and S. Orlov, "Holographic storage dynamics in lithium niobate: theory and experiment," J. Opt. Soc. Am. B 13, 2513-2523 (1996).

39. W. Philips and D. L. Staebler, "Control of the $\mathrm{Fe}^{2+}$ concentration in iron-doped lithium niobate," J. Electr. Mater. 3, 601-617 (1974).

40. K. Buse, A. Gerwens, S. Wevering, and E. Krätzig, "Chargetransport parameters of photorefractive strontium-barium niobate crystals doped with cerium," J. Opt. Soc. Am. B 15, 1674-1677 (1998) 